\section{GP211 POSITIVE CORRELATION OF SERUM ALKALINE PHOSPHATASE AND SERUM ALANINE TRANSAMINASE WITH THE ABUNDANCE-BASED INDICES OF THE MICROBIOTA IN OBESE ADOLESCENTS}

'Lyubov Rychkova, 'Evgenia Novikova*, ${ }^{1,2}$ Natalia Belkova, 'Anna Pogodina, ${ }^{1}$ Anastasiya Romanitsa, ${ }^{1}$ Tatyana Bairova. ${ }^{1}$ Scientific Centre of Family Health and Human Reproduction Problems, Irkutsk, Russian Federation; ${ }^{2}$ Limnological Institute SB RAS, Irkutsk, Russian Federation

\subsection{6/archdischild-2019-epa.270}

Introduction Obesity is a multifactorial disease with genetic, psychological, nutritional, biochemical, and microbiological causes. In previous studies, obesity has been linked to a different composition of the gut microbiota. The gut microbiome composition might be described by several alpha diversity metrics: OTU (operational taxonomic unit) number, Chao1 and ACE (abundance-based coverage estimators), and Shannon and Simpson indices (richness and evenness estimators). On the other hand, recent studies demonstrate a correlation of elevated serum biochemical markers with obesity. Consequently, the linkage between biochemical markers and diversity indices of gut microbiome in obesity might be important and indicative. So, the aim of this study is to check correlation between alpha diversity metrics and obesity-associated metabolic risk factors, such as serum alanine transaminase (ALT) and serum alkaline phosphatase (AP), at patients with obesity and normal weight.

Methods Sixteen subjects were obese adolescents $\left(\mathrm{BMI}=33.07 \pm 4,03 \mathrm{~kg} / \mathrm{m}^{2}\right), 19$ subjects were healthy adolescents $\left(B M I=20.18 \pm 1,76 \mathrm{~kg} / \mathrm{m}^{2}\right)$. These two groups were comparable by ethnicity (100\% participants are Caucasians), gender (20 males, 15 females), and age (mean age 14.7 \pm 1.69 years).

Metagenome sequencing of V3-V4 regions of $16 \mathrm{~S}$ rDNA, raw data analysis, OTU clustering, species annotation, and estimation of alpha diversity indices were done by Novogene Company (China). Concentration of serum ALT and AP were measured using Mindray Automatic Biochemistry Analyzer. Data were analyzed using the STATISTICA 8.0 software package. Statistical significance was accepted at the $\mathrm{p}<0.05$ level.

Results AP data from 11 obese patients, and 7 adolescents with normal weight were included in this study. ALT and OTU number, Chao1, ACE, Shannon and Simpson indices were measured for all respondents $(n=35)$. The WilcoxonMann-Whitney test didn't show significant differences between the obesity and control groups, despite the fact that alpha diversity metrics and AP were higher in the control group $(p>0.05)$. However, ALT was higher, but not significant in the obesity group $(\mathrm{p}>0.05)$.

Spearman's rank correlation coefficient showed significant correlations for AP and ACE $\square$ Chao1 ( $r s=0.93)$ in the obesity group, but not with Shannon and Simpson indices ( $r s=0.26)$. The strong positive correlation also were observed for AP and OTU number $(\mathrm{rs}=0.85)$. Either, we observed small, but significant correlations between ALT and ACE, Chao1 $\square$ OTU number ( $r=0.4)$. There were no significant relationships between ALT and AP with alpha diversity metrics in the control group ( $r s \leq 0.3$ ).

Conclusion The study shows a positive relationship between alpha diversity metrics of intestinal microbiota and serum AP and ALT in adolescents with obesity.

\section{GP212 22Q11 DELETION SYNDROME IN IRELAND - REVIEW OF MENTAL HEALTH NEEDS IN CHILDREN FROM A NEWLY ESTABLISHED CLINIC}

${ }^{1}$ Siobhan McCormack*, ${ }^{2}$ Vaselina Gadancheva, ${ }^{3,4}$ Lorna Kerin, ${ }^{1}$ Suzanne Kelleher,

${ }^{2,3}$ Fiona McNicholas. 'Department of General Paediatrics, Our Lady's Children's Hospital, Crumlin, Dublin, Ireland; 'Department of Child Psychiatry, Our Lady's Children's Hospital, Crumlin, Dublin, Ireland; ${ }^{3}$ Department of Child and Adolescent Psychiatry, University College Dublin, Dublin, Ireland; ${ }^{4}$ Tusla Child and Family agency, Dublin, Ireland

10.1136/archdischild-2019-epa.271

Background 22q11 Deletion Syndrome is a complex, multi-system disorder with a prevalence of 1 in 2000 to 4000 people. Affected children may present with a diverse range of medical conditions and they are extremely vulnerable to psychiatric illness throughout their lifespan. This population are widely known to be at risk of developing psychotic illness with $10 \%$ of adolescents and over $25 \%$ of adults with $22 \mathrm{q}$ developing psychotic disorders. Studies have also shown that up to $57.7 \%$ experience social anxiety and up to $50 \%$ are diagnosed with Autistic Spectrum Disorder.

Aims To review the mental health needs of children with $22 \mathrm{qDS}$ at the time of their first dedicated tertiary clinic review and establish if a dedicated clinic can identify and address unmet needs within this population.

Methods Retrospective audit of outcomes of all first clinic appointments since the establishment of the new dedicated 22qDS paediatric clinic from October 2017 to January 2019. Previous mental health service input along with referrals made were recorded.

Results In the first 14 months of the 22q11DS clinic 43 children were assessed. 11 children (26\%) had been seen by mental health services previously. Of those, 5(45\%) children had anxiety, 1(9\%) ADHD, 1(9\%) both anxiety and ADHD, 1 $(10 \%)$ both anxiety and autism, 2(\%) not specified and 1(9\%) had a normal assessment.

Overall 22(48\%) children have been referred from the clinic for psychiatric input. 11(24\%) have been seen to date with $55 \%$ of those seen having a diagnosis of anxiety and $27 \%$ a diagnosis of ADHD. A further 11(24\%) children are currently waitlisted for assessment.

Conclusion Significant mental health needs were identified in this group of children. Early assessment and intervention to address these needs at an appropriate time in their development could prevent deterioration and use of crisis and acute services in this vulnerable population and help maintain wellbeing and quality of life for children and families.

\section{GP213 THE EFFECT OF STRUCTURED PYHSICAL ACTIVITY ON SLEEP AND MENTAL HEALTH IN SEVERE AUTISTIC CHILDREN}

${ }^{1}$ Tulin Fidan*, 'Bengisu Asfuroglu, ${ }^{2}$ Mehmet Yanardag. ${ }^{1}$ Eskisehir Osmangazi University Faculty of Medicine, Eskisehir, Turkey; ${ }^{2}$ Anadolu University Institute for Disabled Individuals, Eskisehir, Turkey

\subsection{6/archdischild-2019-epa.272}

Introduction Autism Spectrum Disorder (ASD) is a neurodevelopmental disorder characterizing deficits in social communication skills and restricted repertoire of interests, behaviors and atypical sensory reactivity(APA 2013).Children with ASD suffer from sleep problems, particularly insomnia, at a higher rate than typically developing children (Cortesi $\mathrm{F}$ et al,2010).It is 
known that sleep disturbances cause behavioral, affective and cognitive mental health problems. In this study we investigate the effect of structured pyhsical activity on sleep and mental health in severe autistic children.

Methods Thirty-six children who have severe autistic disorder according to Children's Autism Rating Scale participiated the study.Structured pyhsical exercise applied 6 months one by one all these children.Before beginning the physical exercise programme and after the programme we evaluated autistic children with Aberrant Behavior Checklist(ABC), The Children's Sleep Habits Questionnaire(CSHQ).SPSS 18.0 was used for statistical analyses.

Results The children with ASD were between 7-17 years old and the mean age was $12,5 \pm 2,1$ years old. There was significant difference in total scores of $\mathrm{ABC}$ before and after structured physical activity $(\mathrm{p}=0.05, \mathrm{z}=-1.918)$. The mean scores of Hyperactivity-Non compliance subscale were declined significantly after physical activity $(\mathrm{p}=0.045, \mathrm{z}=-2.000)$.

The rate of clinically significant sleep problems was $80.6 \%$ before activity and the rate was $66.7 \%$ after activity. There were statistically significant differences in total CSHQ scores before and after structured physical activity $(p=0.01, z=-3.470)$. The mean scores of Bedtime Resistance $(\mathrm{p}=0.01 \mathrm{z}=-3.128)$ and parasomnias subscale $(\mathrm{p}=0.29, \mathrm{z}=-$ 2187) were declined statistically significant after physical activity.

Discussion In this study the rate of sleep problems is very high about $80 \%$ among severe autistic children as similar to literature(Cortesi $\mathrm{F}$ et al,2010). We found that sleep resistance and parasomnias were declined after phycical activity. Also the hyperactivity behaviors get better after activity.Tatsumi et al (2015) found associations between phycical activity and the quality of sleep were different depending on when the PA occurred. High levels of morning and afternoon activity were associated with early sleep onset among ASD children. Highlevel morning and afternoon activity should be encouraged to improve the sleep quality of children with ASD.Identifying and treating sleep disorders may result not only in improved sleep, but also impact favorably on daytime behavior and family functioning(Cortesi $\mathrm{F}$ et al,2010).

Future studies are needed have larger groups and control subjects must be done to clarify the positive effect of activity on sleep and behavior.

\section{GP214 INCREASED MARKER OF ENDOTHELIAL CELL DYSFUNCTION SVCAM-1 IN UMBILICAL CORD BLOOD IN NEONATES BORN TO OBESE WOMEN}

Valeria Novikova*, Dmitriy Ivanov, Yuriy Petrenko, Aleksey Yakovlev, Olga Gurina, Olga Varlamova, Aleksey Blinov. St. Petersburg State Pediatric Medical University, St. Petersburg, Russian Federation

10.1136/archdischild-2019-epa.273

Introduction Increased level of angiogenic and adhesion molecules released from adipose tissue in pregnant women with obesity may affect the process of angiogenesis, metabolic state and inflammatory potential in both mother and her offspring. Objectives To determine the effect of maternal obesity on the level of sVCAM-1 and VEGF-A in mothers and their newborn babies.

Methods We conducted a prospective, observational study with the inclusion of 42 mothers and their newborn babies. Main group included 21 mothers with obesity and their newborn babies. Control group consisted of 22 women with normal body mass index (BMI) before and during pregnancies and their newborn babies. We analyzed serum concentrations of vascular cell adhesion molecule 1 (sVCAM-1) and vascular endothelial growth factor A (VEGF-A) in maternal cord blood and in venous blood of newborn babies in 2 day after birth. The normal level of sVCAM-1 for adults is 400.6--1340.8 ng/ $\mathrm{ml}$; VEGF-A (for adults) - 0-42.6 pg/ml. Data was analyzed using the statistical package Statistica 10.0 for Windows-10. The significance of the differences was determined at $\mathrm{P}$ value $<0.05$.

Results The level of markers of endothelial dysfunction in cord blood exceeded normal values in all examined women. Concentration of sVCAM-1 in maternal cord blood was significantly higher in women with obesity compare to women with normal BMI $(4926.19 \pm 1158.63 \mathrm{ng} / \mathrm{ml}$ vs $3294.66 \pm 1338.23$ $\mathrm{ng} / \mathrm{ml}, \mathrm{p}<0,0001)$. Concentration of VEGF-A in maternal cord blood in both groups $(224.74 \pm 57.41 \mathrm{pg} / \mathrm{ml}$ and 197.29 $\pm 180.44 \mathrm{pg} / \mathrm{ml} ; \mathrm{p}>0.05$ ), concentration of sVCAM-1 in newborns serum in both groups $(4019.32 \pm 1024.45 \mathrm{ng} / \mathrm{ml}$ and $4300.35 \pm 948.08 \mathrm{ng} / \mathrm{ml}, \mathrm{p}>0.05)$ and concentration of VEGF$\mathrm{A}$ in newborns serum in both groups $(208.42 \pm 141.72 \mathrm{pg} / \mathrm{ml}$ and $170.53 \pm 152.64 \mathrm{pg} / \mathrm{ml} ; \mathrm{p}>0.05)$ had no significant differences. The level of sVCAM-1 in cord blood was higher than in newborns serum in main group (4926.19 \pm 1158.63 and $4019.32 \pm 1024.45, \mathrm{p}<0.05)$, but lower than in the control group (3294.66 $\pm 1338.23 \square 4300.35 \pm 948.08, p<0.05)$. We did not reveal any significant differences in level of VEGF-A in cord blood and in newborns serum in both groups.

Conclusions Pregnancy in obese women associated with chronic endothelial activation secondary to increased production of vascular cell adhesion molecule 1 (sVCAM-1). The level of markers of endothelial cell dysfunction in infants may be associated with a different course of the adaptation period. Pregestational or pregnancy associated disorders, can have a different impact on endothelial function in pregnant women. The dynamics of the endothelial dysfunction markers in umbilical cord blood and serum of infants requires additional study.

\section{GP215 GROWTH AND NUTRITIONAL STATUS IN CHILDREN WITH PRADER WILLI SYNDROME}

${ }^{1}$ Christina Meade*, ${ }^{1}$ Ruth Martin, ${ }^{2}$ Ann McCrann, ${ }^{3}$ Jacqueline Lyons, ${ }^{3,4}$ Edna Roche. ${ }^{1}$ Department of Nutrition and Dietetics. National Children's Hospital, Tallaght University Hospital, Dublin, Ireland; ' $D$ Department of Paediatric Growth Diabetes and Endocrinology, National Children's Hospital, Tallaght University Hospital, Dublin, Ireland; ${ }^{3}$ Department of Paediatric Growth Diabetes and Endocrinology, National Children's Hospital, Tallaght University Hospital, Tallacht, Ireland; ${ }^{4}$ Discipline of Paediatrics, The University of Dublin, Trinity College Dublin, Dublin, Ireland

\subsection{6/archdischild-2019-epa.274}

Objective To explore growth and nutritional status in children with Prader Willi Syndrome (PWS)

Methods All children with a genetically confirmed diagnosis of PWS, attending the National Children's Hospital Tallaght were invited to participate $(n=44)$. Anthropometry, height/length, weight and body mass index (BMI) were measured were appropriate. Body composition was evaluated using bioelectrical impedance analysis. Parents completed a feeding questionnaire to identify early feeding issues in PWS. Parents received training on recording dietary intake before completing 3 Day 